\title{
The Hierarchical Implications of Internet Gaming Disorder Criteria: Which Indicate more Severe Pathology?
}

\author{
Seung-Yup Lee ${ }^{1}$, Hae Kook Lee', Hyunsuk Jeong², Hyeon Woo Yim², Soo-Young Bhang ${ }^{3}$, Sun-Jin Jo², \\ Kyung-Young Baek², Eunjin Kim², Min Seob Kim', Jung-Seok Choi ${ }^{4}$, and Yong-Sil Kweon ${ }^{1}$ \\ 1'Department of Psychiatry, Uijeongbu St. Mary's Hospital, College of Medicine, The Catholic University of Korea, Uijeongbu, Republic of Korea \\ 2Department of Preventive Medicine, College of Medicine, The Catholic University of Korea, Seoul, Republic of Korea \\ ${ }^{3}$ Department of Psychiatry, Eulji Hospital, College of Medicine, Eulji University, Seoul, Republic of Korea \\ ${ }^{4}$ Department of Psychiatry, SMG-SNU Boramae Medical Center, Seoul, Republic of Korea
}

Objective To explore the structure of Internet gaming disorder (IGD) criteria and their distribution according to the different severity level of IGD. The associations of psychiatric comorbidities to each IGD symptom and to the IGD severity were also investigated.

Methods Consecutively recruited 330 Korean middle school students underwent face-to-face diagnostic interviews to assess their gaming problems by clinicians. The psychiatric comorbidities were also evaluated with a semi-structured instrument. The data was analyzed using principal components analysis and the distribution of criteria among different severity groups was visualized by plotting univariate curves.

Results Two principal components of 'Compulsivity' and 'Tolerance' were extracted. 'Decrease in other activities' and 'Jeopardizing relationship/career' may indicate a higher severity of IGD. While 'Craving' deserved more recognition in clinical utility, 'Tolerance' did not demonstrate much difference in distribution by the IGD severity. Internalizing and externalizing psychiatric disorders differed in distribution by the IGD severity.

Conclusion A hierarchic presentation of IGD criteria was revealed. 'Decrease in other activities' and 'Jeopardizing relationship/career' may represent a higher severity, thus indicating more clinical attention to such symptoms. However, 'Tolerance' was not found to be a valid diagnostic criterion.

Psychiatry Investig 2017;14(3):249-259

Key Words Internet gaming disorder, Diagnostic criteria, Severity, Hierarchy, Principal components analysis.

\section{INTRODUCTION}

The widespread usage of Internet brought a lot of convenient changes in daily lives and lessened the effect of physical barriers for communication, bringing the people closer together. On the other hand, concerns have also been raised for the negative psychosocial consequences of the information technologies. ${ }^{1}$ The real-life interactions are increasingly displaced by online-interactions. ${ }^{2}$ Moreover, the unreserved contents of the Internet also raised concerns about its poten-

Received: June 23, 2016 Revised: August 22, 2016

Accepted: September 27, 2016 Available online: May 12, 2017

$\triangle$ Correspondence: Yong-Sil Kweon, MD, PhD

Department of Psychiatry, Uijeongbu St. Mary's Hospital, College of Medicine,

The Catholic University of Korea, 271 Cheonbo-ro, Uijeonbu 11765, Republic of Korea

Tel: +82-31-820-3032, Fax: +82-31-847-3630, E-mail: yskwn@catholic.ac.kr

(a) This is an Open Access article distributed under the terms of the Creative Commons Attribution Non-Commercial License (http://creativecommons.org/licenses/bync/4.0) which permits unrestricted non-commercial use, distribution, and reproduction in any medium, provided the original work is properly cited. tial adverse consequences on mental health. Of particular, Internet gaming has gained growing attention in the field of psychiatry as it is enlisted in the section III of the new diagnostic and statistical manual of mental disorders (DSM) $-5^{3}$ and currently proposed as a formal diagnostic criteria in the International Classification of Diseases (ICD)-11. ${ }^{4}$ IGD further raises concerns in that the problems are anticipated to grow with wider spread of Internet access around the globe and the growing popularity of smartphones. ${ }^{5}$ Therefore, the proposed inclusion of gaming disorder in the ICD-11 seems timely and in right direction.

Previously, the concept of "addiction" was only confined to psychoactive substance. However, "behavioral addiction" became official with the introduction of new category for "nonsubstance-related disorders" in DSM-5 and "disorders due to addictive behaviors" in the ICD-11 beta draft. In contrast to IGD being in the section III of DSM, the ICD-11 is anticipated to include it as a formal diagnostic entity as gaming disorder. 
The concept of two proposed diagnostic criteria are similar in general. However, there are some differences between them. While the DSM-5 stated that non-Internet computerized games could be also be involved for IGD in contradiction to its nomenclature, the ICD-11 classified gaming disorder into online and offline subtypes. ${ }^{3,4}$ However, the DSM-5 introduced other classification by severity; mild, moderate and severe. ${ }^{3}$ Another major distinction between the two diagnostic systems is that the ICD-11 excluded the criterion of 'Tolerance' or 'Withdrawal' as diagnostic criteria unlike the DSM-5. ${ }^{4}$

As stated in the diagnostic criteria of both the DSM-5 and the ICD-11 draft, a significant impairment may occur due to IGD in personal, family, social, school or occupational areas. ${ }^{3,4}$ Vocational and academic problems may also, in turn, exert negative impacts in mental health..$^{6-8}$ There is now a growing consensus that IGD is associated with increased psychological distress and psychiatric comorbidities such as depression, anxiety disorders, sleep problems and attention-deficit hyperactivity disorder (ADHD) and etc. ${ }^{9-13}$ However, as IGD is a novel diagnostic entity, its natural course and causality with psychiatric comorbidities are not well understood. Furthermore, the structural component and inter-relationships between the major IGD symptoms remain unclear. In addition, basic epidemiological data like the IGD prevalence greatly vary across the literatures $(1.5-50 \%){ }^{14-16}$

Such large variation in prevalence may have been resulted from different definition of IGD due to lack of gold standard or due to differences in environmental factors like socio-cultural attitudes towards gaming, difference in high-speed Internet accessibility, smartphone usage rates and so on. However, we believe that the methodological limitations may also contribute to such great variation. In general, on-line surveys demonstrated higher life prevalence (3.4-50\%) for IGD while written surveys displayed somewhat lower life prevalence (1.5-9.9\%). ${ }^{16}$ Such phenomena may stem from sampling bias, where more problematic subjects could have been recruited in online surveys. In addition to potential errors in sampling, all such researches utilized surveys to estimate prevalence of IGD. Data collection through self-reports can be convenient and inexpensive. However, reliance on selfmeasurement encompass major limitations such as errors arising due surveying attitude or sincerity; subjectivity (i.e., different values and thresholds to gaming and its problem severity); false or concealing responses leading to underestimation or different levels of understanding to the questionnaires. ${ }^{17,18}$ In order to overcome the aforementioned methodological limitations, we collected data by conducting a large scale face to face diagnostic interviews by clinicians.

This study's main objectives were to explore the structural component of IGD criteria and investigate whether they have a hierarchic order. The research conducted by Toce-Gerstein et al. ${ }^{19}$ on gambling disorder provided an important insight on the relationships of gambling symptoms across the different severity groups. However, the data was derived from mixed samples and even though it utilized principal components analysis (PCA), not much information regarding the structural component was provided. We believe that PCA can be a particularly valuable method to a novel diagnostic entity like IGD since it does not require any particular structural modeling. To the best of our knowledge, there were two recent researches that analyzed gamers with PCA..$^{20,21}$ However, they displayed the methodological limitations described above, which were online sample recruitment through game forums and lacking objective measurements by reliance on self-reports. One research utilized PCA to analyze structure for new video gaming instrument and found two components corresponding to 'impaired control' and 'negative consequences', however the results could had been confounded by the gender effect since the sample was predominated by males. ${ }^{21}$ Furthermore, the sample of other study was comprised only by a particular game of 'World of Warcraft' in one study, thus limiting its generalizability to other games or game genres. ${ }^{20}$

Exploring the structural component of IGD criteria may provide a valuable answer to what dimensions the IGD criteria are actually measuring and how the symptoms are related to each other. Further investigating the relationships between the IGD symptoms and their relevance to psychiatric comorbidity could provide more detailed information on its nature. The positive proportions of each IGD criterion among different severity levels were compared to observe which criteria reveal more severe form of IGD pathology.

\section{METHODS}

\section{Participants and procedures}

The study was carried out as a part of the Internet user Cohort for Unbiased Recognition of gaming disorder in Early adolescents (iCURE) study on Internet game and smart phone addiction (clinicaltrials.go videntifier: NCT 02415322). The iCURE is a prospective cohort study, carefully designed to identify risk and protective factors for IGD and Social Networking Service (SNS) addiction and their natural courses, especially in relation to psychiatric disorders.

The participants were first year middle school students in Seoul metropolitan area in the Republic of Korea. They were consecutively enrolled from 15th of September to the end of October in 2015. After obtaining permissions from the adolescents and their parents/guardians, baseline socio-demographic factors, Internet usage and gaming related factors were collected through questionnaires. Of total of 330 students as- 
sessed, the number of males was $163(49.4 \%)$ and the number of females was 167 (50.6\%). A major potential limitation of school survey is the error originated by giving out false answers in an attempt to conceal the students' own problems. Distributing and collecting paper survey may heighten up the concern of the surveyees about teacher or parents finding out about their problematic behaviors. Thus, self-measurements were collected through the study's designated website (http:// cohort.co.kr). After logging into our website with previously provided unique authentication codes, each participant completed the web-based survey.

Every enrolled participant also underwent face-to-face diagnostic interviews with clinicians. The diagnostic interviews took place in the participated schools a week after the baseline study completion. The students were assessed for IGD according to the DSM-5 IGD criteria plus for craving symptoms. A semi-structured interview was also administered to explore any presence of psychiatric disorders. This study was approved by the Institutional Review Board of The Catholic University of Korea (MC140NM10085) and was conducted in accordance with the principles of Declaration of Helsinki.

\section{Measures}

For the objective measurement of the Internet gaming problems, students were assessed according to the DSM-5 IGD criteria. In addition, craving was also assessed during the interview as it was not measured in the newly proposed DSM-5 IGD criteria. Craving was evaluated by asking the following questions - "Do you frequently experience a strong urge for gaming?", "Once thought about gaming comes into your mind, is it difficult to suppress such desire?" For psychiatric comorbidity, each student also underwent a semi-structured interview using Kiddie-Schedule for Affective Disorders and Schizophrenia-Present and Lifetime Version-Korean version (K-SADS), which had been validated previously. ${ }^{22}$

\section{Interviewers}

The interviewer pool was comprised of 9 psychiatrists and one clinical psychologist, whose working experiences were at least three years in clinical practice. Every interviewer was intensively pre-trained by both addiction specialists and children-adolescents psychiatry specialists. The training course included instruction on the assessing instruments; diagnostic considerations; severity judgments; general techniques of interviewing children and adolescents and techniques for probing and clarifying ambiguous answers.

\section{Statistical analysis}

A PCA was performed to explore major components of the proposed DSM- 5 criteria of IGD and craving for Internet gaming. PCA extracts components based on variance in a way that maximize the overall variance. PCA was chosen over factor analysis because it does not require any modeling, which encompasses presumptions on relationships between factors. Since IGD is rather a novel diagnostic entity, the relationship between different IGD symptoms remains unclear. Therefore, it was considered that presuming any particular structures may lead to unnecessary biases. Components yielding eigenvalue greater than 1.0 were included.

Next, the relative proportion of each IGD symptom was explored according to the IGD severity. To determine of which symptoms are more endorsed by the increasing severity of IGD, positive endorsers were grouped by the total number of endorsed IGD domains that ranged from 1 to 8 . The proposed IGD criteria suggested five or more criteria to be met to diagnose IGD in the DSM-5. Therefore, the endorsers were divided into 'subclinical gamers' and 'addicted gamers' by 5 . The subclinical gamers were then again bisected into mild (positive on 1 to 2 numbers of domains) and moderate risk gamers (positive on 3 to 4 numbers of domains). The IGD subjects were also further divided to 'plain' addicted group (positive on 5 to 6 numbers of domains) and 'severe' addicted group (positive on 7 to 8 numbers of domains). As a result, severity grouping were done into four groups with equal interval in terms of counts of positive domains. As well as examining the symptomatic distribution across different severity of IGD, the presence of psychiatric comorbidity was also explored between those four groups. Pairwise comparisons were performed between the adjacent groups using chi-squared or Fisher's exact test with two-tailed statistical significance of 0.05 . The adjacent groups were compared to investigate the hierarchical order of each symptom by different severity.

To further determine the distribution of each symptom according to severity, univariate curves were plotted across every IGD symptoms. For each IGD symptom, the endorsement rate of a particular symptom was plotted against the whole positive endorsers for four different severity groups. The goodness of fit for each symptom curve was tested by calculating the squared correlation $\left(R^{2}\right)$. Whether linear or polynomial, the best fitting curve was plotted to maximize the $R^{2}$ between the actual values and the values predicted by the curve. As previously explained by Toce-Gerstein et al., ${ }^{19}$ adecelerating curvilinear line concaving downwards indicates 'low-threshold' meaning the symptom is more prevalent in ones with low severity. On the other hand, an accelerating curvilinear line concaving upwards indicates 'high-threshold' meaning the symptom is more prevalent in ones with high severity.

Finally, the relationships of ten symptoms, with DSM-5 IGD criteria and craving combined, were additionally investigated by Cramer's Vassociations $(\phi)$. Firstly, the pair wise 
associations of each criterion were computed to examine how the factors within the same component of PCA were correlated. Secondly, their associations with psychiatric comorbidities were also computed. Exploring the associations of each IGD criterion to psychiatric comorbidities may provide valuable clues to of which IGD symptoms are influenced by comorbid psychiatric conditions or vice versa. However, the wide spectrum of psychiatric comorbidities could exert different effects to a particular clinical manifestation of IGD. Thus, the mixed effects may, in turn, cancel out the psychiatric influences on IGD symptoms when the psychiatric comorbidities are treated as a whole. Krueger previously suggested two factors for mental disorders; internalizing and externalizing. ${ }^{23}$ Many researches adopted this concept of clustered correlated syndromes that grouped depression and anxiety disorders into internalizing and grouping $\mathrm{ADHD}$, antisocial, conduct disorders or substance disorders as externalizing behavioral problems. ${ }^{24-27}$ Therefore, we further bisected the psychiatric comorbidities into internalizing (depression, anxiety and adjustment disorders) and externalizing (ADHD, ODD, conduct disorder and tic disorder) group.

All statistical analyses were performed using the software package SAS Enterprise Guide 7.1 (SAS Institute, Inc, Cary, North Carolina) or R software version 2.15.3 (R Foundation for Statistical Computing, Vienna, Austria; www.r-project.org).

\section{RESULTS}

\section{Basic clinco-demographics}

The clinco-demographic characteristics are shown in Table 1. It can be seen that the gender ratio of our sample is well balanced. For psychiatric comorbidities, 21 students had mood disorders, which were adjustment disorder with depressed mood, unipolar or bipolar depression. Twelve students were diagnosed for any kind of anxiety disorders. By avoiding multiple-counting of the ones with multiple disorders, the net total of the mood and anxiety disorders yielded 28 (8.5\%) and they were categorized as internalizing disorders. On contrary, following disorders were categorized as externalizing disorders: ADHD, oppositional defiant disorder (ODD), Conduct disorder and tic disorder. Eleven students had ADHD. There were two students having a Tic disorder. Three students were diagnosed with oppositional defiant disorder (ODD) or Conduct disorder, with two students also having a mood disorder. Yet,

Table 1. Clinico-demographic characteristics of interviewees $(\mathrm{N}=330)$

\begin{tabular}{|c|c|c|}
\hline & $\mathrm{N}$ & $\%$ \\
\hline \multicolumn{3}{|l|}{ Gender } \\
\hline Male & 163 & 49.4 \\
\hline Female & 167 & 50.6 \\
\hline \multicolumn{3}{|l|}{ Psychiatric comorbidity } \\
\hline No & 288 & 87.3 \\
\hline Yes & 42 & 12.7 \\
\hline Internalizing disorders & 28 & 8.5 \\
\hline Mood disorders & 21 & 6.4 \\
\hline Anxiety disorders & 12 & 3.6 \\
\hline Externalizing disorders & 13 & 3.9 \\
\hline ADHD & 11 & 3.4 \\
\hline ODD or conduct disorder & 3 & 0.9 \\
\hline Tic disorder & 2 & 0.6 \\
\hline Psychotic disorder NOS & 1 & 0.3 \\
\hline \multicolumn{3}{|l|}{ Game playing } \\
\hline Unknown & 1 & 0.3 \\
\hline No & 71 & 21.5 \\
\hline Yes & 258 & 78.2 \\
\hline Daily playing time (minute) $(\mathrm{N}=258)$ & Mean (SD) & Median (min-max) \\
\hline Weekday & $119.0(117.9)$ & $90.0(0.0-730.0)$ \\
\hline Weekend & $207.5(189.8)$ & $180.0(0.0-1440.0)$ \\
\hline Average (weekday+weekend) & $144.3(126.1)$ & $107.1(1.4-925.7)$ \\
\hline
\end{tabular}

ADHD: attention-deficit hyperactivity disorder, ODD: oppositional defiant disorder, NOS: not otherwise specified 
Table 2. Summary of principal component analysis $(\mathrm{N}=69)$

\begin{tabular}{|c|c|c|c|c|c|c|}
\hline $\begin{array}{c}\text { Principal } \\
\text { component }\end{array}$ & Eigenvalue & Proportion & $\begin{array}{l}\text { Cumulative } \\
\text { proportion }\end{array}$ & IGD symptoms & $\begin{array}{l}\text { Factor } 1 \\
\text { loading }\end{array}$ & $\begin{array}{l}\text { Factor } 2 \\
\text { loading }\end{array}$ \\
\hline 1 & 3.97 & 0.40 & 0.40 & Preoccupation & 0.69 & 0.20 \\
\hline 2 & 1.09 & 0.11 & 0.51 & Withdrawal & 0.62 & -0.15 \\
\hline 3 & 0.93 & 0.09 & 0.60 & Tolerance & 0.38 & 0.77 \\
\hline 4 & 0.81 & 0.08 & 0.68 & Loss of control & 0.71 & 0.31 \\
\hline 5 & 0.72 & 0.07 & 0.75 & Decrease in other activities & 0.70 & -0.45 \\
\hline 6 & 0.66 & 0.07 & 0.82 & Persistent use despite negative results & 0.70 & 0.06 \\
\hline 7 & 0.62 & 0.06 & 0.88 & Deceiving & 0.57 & -0.06 \\
\hline 8 & 0.48 & 0.05 & 0.93 & Escapism & 0.52 & -0.05 \\
\hline 9 & 0.37 & 0.04 & 0.96 & Jeopardizing relationship/career & 0.64 & -0.36 \\
\hline 10 & 0.36 & 0.04 & 1.00 & Craving & 0.67 & 0.02 \\
\hline
\end{tabular}

IGD: internet gaming disorder

those two were categorized as externalizing disorders considering the dominant clinical feature in them. Altogether, the net total of externalizing disorders was 13 (3.9\%).

While 71 students $(21.5 \%)$ were non-gamers, most of the students ( $n=258,78.2 \%)$ played Internet game by either personal computers or smartphones. The mean gaming time for weekday and weekend were 119.0 and 207.5 minutes, respectively. The players spent 144.3 minutes daily for gaming on average.

\section{Principal components analysis}

Through PCS, two components were found to have Eigen value greater than 1.0. The first component demonstrated Eigen value of 3.97 and was comprised by symptoms other than tolerance. The second component displayed Eigen value of 1.09 and included only one symptom of tolerance. Altogether, the two components' cumulative percentage of explained variance was $51 \%$ (Table 2).

The first component explained 40 percentage of the variance and was loaded by nine symptoms. The factor loadings for the first component ranged from 0.52 to 0.71 , thus all symptoms were included. The factor loadings of each are as following in descending order: 'Loss of Control' (0.71), 'Persistent use despite Negative results' (0.70), 'Decrease in other activities' (0.70), 'Preoccupation' (0.69), 'Craving' (0.67), 'Jeopardizing relationship/career' (0.64), 'Withdrawal' (0.62), 'Deceiving' (0.57), and 'Escapism' (0.52). To put into words, the component is represented by 'loss of control for gaming while being preoccupied and craving to play instead of all other activities and despite negative consequences.' In medical subject headings provided by United States National Library of Medicine, compulsive behavior is 'the behavior of performing an act persistently and repetitively without it leading to reward or pleasure.28 Thus, compulsivity was considered to provide the best fitting explanation for this component of 'losing control over gaming and prioritiz-

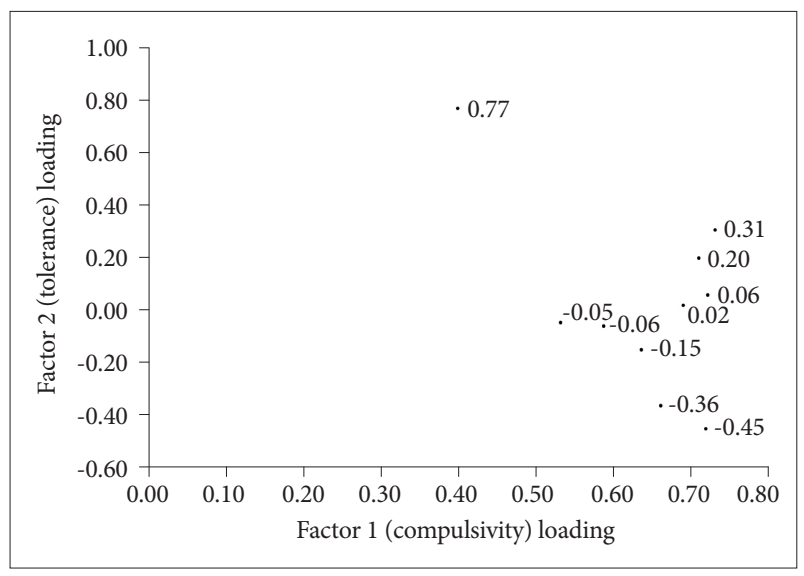

Figure 1. Biplot of the two Principal Components.

ing the repetitive behavior of gaming over other activities despite harms. Thus, the first principal component was labeled as compulsivity.

The second component explained 11 percentage of the variance. The symptom that showed the highest factor loading was tolerance with factor loading of 0.77 . However, all other symptoms did not demonstrate meaningful factor loadings in the second principal component. Bi-plotting the two principal components revealed the relationships between factors more apparent (Figure 1). While all other symptoms were clustered and demonstrated heavy loadings for the first principal component (compulsivity), the 'Tolerance' criterion displayed a high loading on its own, located aloof from the rest. Although 'Loss of Control' was the factor with second largest loading (0.31) for the second principal component (tolerance), it was closely clustered to the compulsivity component.

\section{Distribution of IGD symptoms and their univariate curves across different severity}

Among the gamers, 69 students (20.9\%) were assessed to be positive on any of the criteria for IGD and craving. Thirty-two 
(9.7\%) and twenty-one (6.4\%) students were positive for 1-2 and 3-4 IGD symptoms, respectively. Sixteen students (4.9\%) demonstrated more than five of the symptoms. When examining the whole positive sample, the most common symptom was 'Loss of Control' (50.7\%) and was followed after by 'Preoccupation' (43.5\%) and 'Craving' (43.5\%). Overall, 'Escapism' (36.2\%) and 'Persistent use despite Negative results' (33.3\%) were also frequently appeared symptoms. Yet, 'Escapism' was more prevalent in low severity group ( $28.1 \%$ vs. $9.4 \%$ of 'Persistent use despite Negative results' in 1-2 positive group), whereas 'Persistent use despite Negative results' were more frequent in higher severity group (100\% vs. $75 \%$ of 'Escapism' in 7-8 positive group) (Table 3 ).

The pairwise Fisher's exact test revealed that three symptoms differed significantly between the mild risk group and the moderate risk group by statistics $(\mathrm{p}<0.05)$. The three symptoms of 'Persistent use despite Negative results' (9.4\% vs. $42.9 \%$ ), 'Jeopardizing relationship/career' (0\% vs. $19.1 \%$ ) and 'Craving' (25.0\% vs. $52.4 \%$ ) were more present in the moderate risk group, who were assessed to have 3-4 IGD symptoms. There were trends that 'Withdrawal' (3.1\% vs. $19.1 \%, \mathrm{p}=0.07$ ) and 'Deceiving' ( $3.1 \%$ vs. $19.1 \%, \mathrm{p}=0.07$ ) were more likely to be displayed by the moderate risk group.

The addicted group differed significantly from the moderate risk group in single symptom of 'Decrease in other activities' ( $14.3 \%$ vs. $50.0 \%, \mathrm{p}<0.05)$. Even though not reaching a statistically significant level, 'Withdrawal' ( $19.1 \%$ vs. $58.3 \%$, $\mathrm{p}=0.05$ ) was again more likely to be observed in the higher adjacent group.

Compared to the addicted group, the 'Jeopardizing rela- tionship/career' symptom tended to be more prevalent in the severe addicted group ( $33.3 \%$ vs. $100 \%$, $\mathrm{p}=0.07)$. However, no statistically significant difference was demonstrated between the addicted group and the severe addicted group (Figure 2).

Upon inspecting the univariate curves plotted for each IGD symptom, it became clearer that the distribution of IGD symptoms varied according to the severity. The symptoms with decelerating curvilinear lines were 'Preoccupation,' 'Withdrawal', 'Deceiving' and 'Tolerance.' However, the shape of the best fitting polynominal regression curve plotted by the criterion of 'Tolerance' was close to 'flat' rather than deceleration. On the other hand, accelerating curvilinear lines were displayed by two symptoms. Those were 'Escapism' and 'Jeopardizing relationship/career.' The rest of the symptoms showed linear relationships in regression curves (Figure 2).

Out of all 69 participants, who were evaluated positive to any of the criteria for IGD and craving, nine students (13.0\%) had the internalizing disorders while five students (7.3\%) had the externalizing disorders. These figures are somewhat higher than the above-mentioned psychiatric comorbidity of the whole sample; 28 (8.5\%) and 13 (3.9\%) for the internalizing and the externalizing disorders, respectively. Although not statistically significant, the psychiatric comorbidities showed an increasing trend as the severity of gaming problem increased. When plotted in unvariate curves, the externalizing disorders demonstrated a decelerating curvilinear line while an accelerating curvilinear line was plotted for the internalizing disorders (Figure 3).

Table 3. Pairwise comparison of each IGD symptom between the adjacent severity groups $(\mathrm{N}=69)$

\begin{tabular}{lccccc}
\hline & Overall (1-8) & Mild risk (1-2) & Moderate risk (3-4) & Addicted (5-6) & Severe addicted (7-8) \\
\hline Students positive to any criteria, N (\%) & $69(20.9)$ & $32(9.7)$ & $21(6.4)$ & $12(3.6)$ & $4(1.2)$ \\
Preoccupation & $30(43.5)$ & $8(25.0)$ & $10(47.6)$ & $9(75.0)$ & $3(75.0)$ \\
Withdrawal & $14(20.3)$ & $1(3.1)$ & $4(19.1)$ & $7(58.3)$ & $2(50.0)$ \\
Tolerance & $14(20.3)$ & $4(12.5)$ & $6(28.6)$ & $3(25.0)$ & $1(25.0)$ \\
Loss of control & $35(50.7)$ & $11(34.4)$ & $10(47.6)$ & $10(83.3)$ & $4(100.0)$ \\
Decrease in other activities & $14(20.3)$ & $1(3.1)$ & $3(14.3)^{\dagger}$ & $6(50.0)^{\dagger}$ & $4(100.0)$ \\
Persistent use despite negative results & $23(33.3)$ & $3(9.4)^{*}$ & $9(42.9)^{*}$ & $7(58.3)$ & $4(100.0)$ \\
Deceiving & $13(18.8)$ & $1(3.1)$ & $4(19.1)$ & $6(50.0)$ & $2(50.0)$ \\
Escapism & $25(36.2)$ & $9(28.1)$ & $8(38.1)$ & $5(41.7)$ & $3(75.0)$ \\
Jeopardizing relationship/career & $12(17.4)$ & $0(0.0)^{*}$ & $4(19.1)^{*}$ & $4(33.3)$ & $4(100.0)$ \\
Craving & $30(43.5)$ & $8(25.0)^{*}$ & $11(52.4)^{*}$ & $8(66.7)$ & $3(75.0)$ \\
Total psychiatric comorbidity & $14(20.3)$ & $5(15.6)$ & $3(14.3)$ & $4(33.3)$ & $2(50.0)$ \\
Internalizing disorders & $9(13.0)$ & $4(12.5)$ & $2(9.5)$ & $1(8.3)$ & $2(50.0)$ \\
Externalizing disorders & $5(7.3)$ & $1(3.1)$ & $1(4.8)$ & $3(25.0)$ & $0(0.0)$
\end{tabular}

All adjacent groups were compared using either chi-squared or Fisher's exact test. ${ }^{*} \mathrm{p}<0.05$ compared between mild and moderate risk groups, ${ }^{\dagger} \mathrm{p}<0.05$ compared between moderate risk and addicted groups, ${ }^{\ddagger}$ number of positive IGD symptoms. IGD: internet gaming disorder 

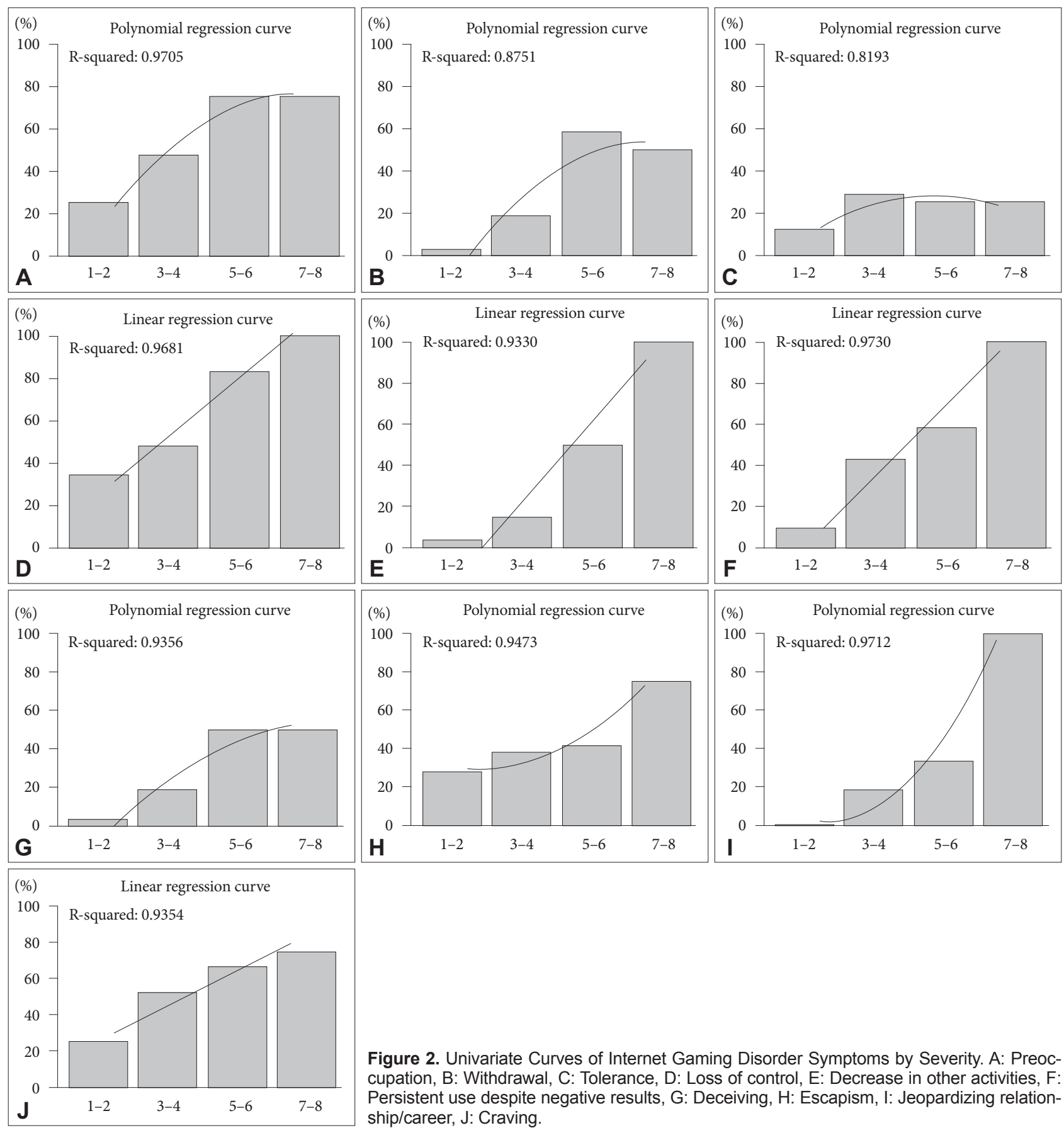

Figure 2. Univariate Curves of Internet Gaming Disorder Symptoms by Severity. A: Preoccupation, B: Withdrawal, C: Tolerance, D: Loss of control, E: Decrease in other activities, F: Persistent use despite negative results, G: Deceiving, H: Escapism, I: Jeopardizing relationship/career, J: Craving.
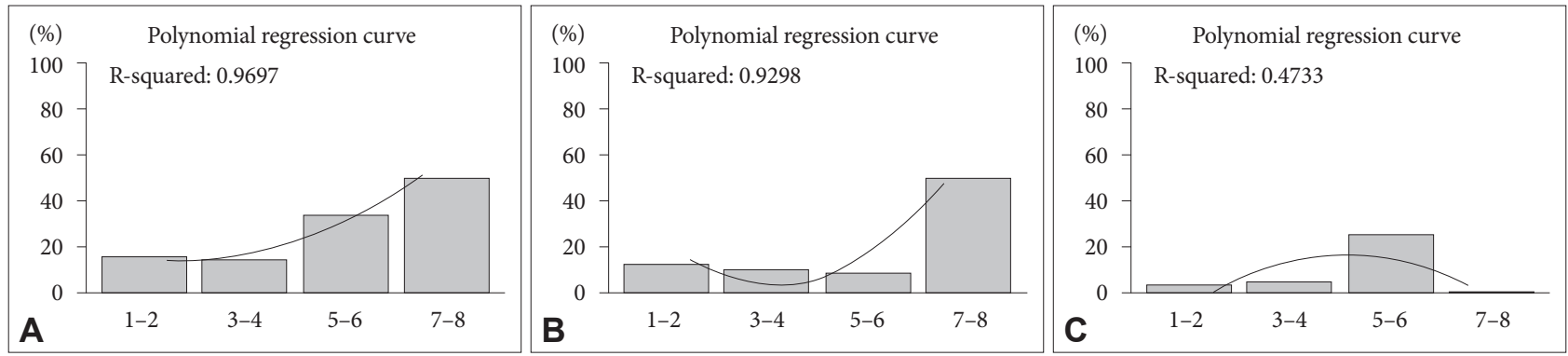

Figure 3. Univariate Curves of Comorbid Psychiatric Disorders by Severity. A: Overall Psychiatric comorbidities, B: Comorbid Internalizing disorders, C: Comorbid Externalizing disorders. 


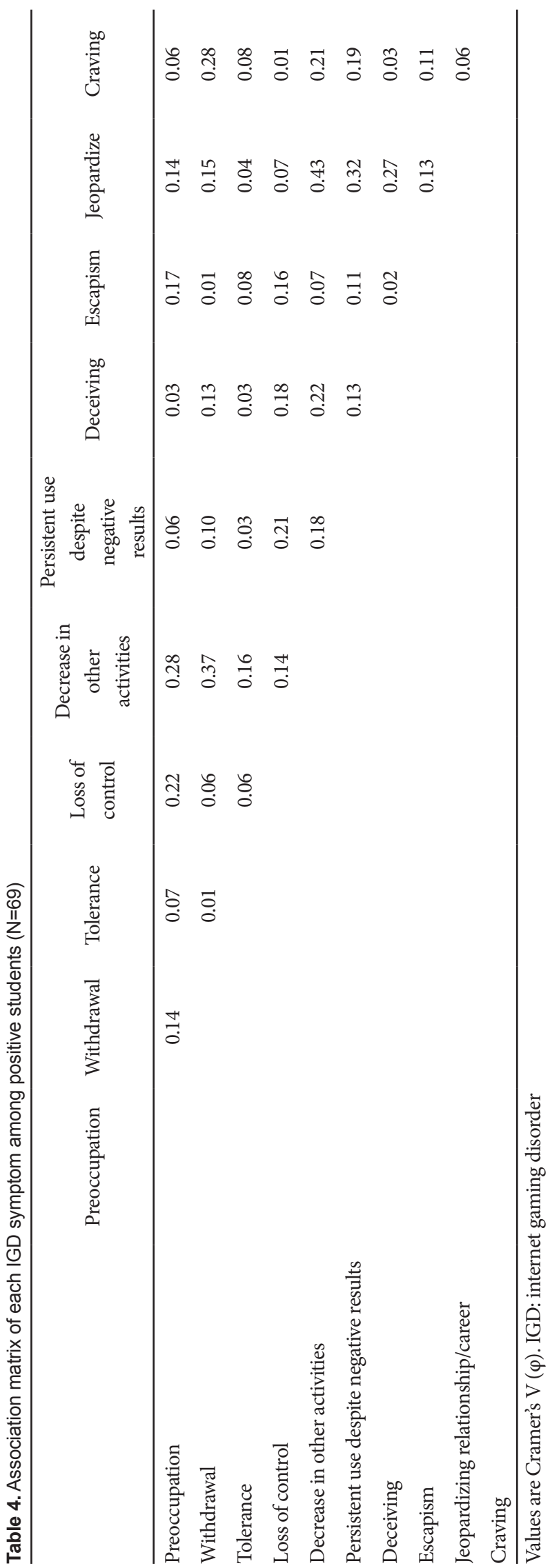

\section{The Pairwise Associations between IGD criteria}

'Preoccupation' showed moderately strong association with 'Decrease in other activities' $(\phi=0.28)$ and moderate association with 'Loss of Control' ( $\phi=0.22)$. While 'Loss of Control' only had moderate association with 'Persistent use despite Negative results' ( $\phi=0.21)$, 'Decrease in other activities' was associated with the most numerous symptoms.

'Decrease in other activities' demonstrated the strongest association with 'Jeopardizing relationship/career' ( $\phi=0.43)$, very strong association with 'Withdrawal' $(\phi=0.37)$ and moderate associations with both 'Deceiving' $(\phi=0.22)$ and 'Craving' $(\phi=0.21)$ (Table 4).

In addition of being associated with 'Loss of Control' ( $\phi=$ 0.22 ), 'Persistent use despite Negative results' was also very strongly associated with 'Jeopardizing relationship/career' $(\phi=0.32)$. Apart from 'Decrease in other activities' $(\phi=0.43)$ and 'Persistent use despite Negative results' $(\phi=0.32)$, 'Jeopardizing relationship/career' also demonstrated moderately strong association with 'Deceiving' ( $\phi=0.27)$.

As well as 'Decrease in other activities' $(\phi=0.37)$, 'Withdrawal' showed moderately strong association with 'Craving' ( $\phi=$ 0.28 ). On the other hand, 'Escapism' did not display much association with the rest of IGD symptoms, though it belonged to the same first component in the PCA. The 'Tolerance' criterion, which comprised the second component in the PCA, also revealed no meaningful associations with the rest of the IGD symptoms (Table 4 ).

The associations between the IGD symptoms and the psychiatric comorbidities were also explored. Three symptoms of 'Preoccupation,' 'Withdrawal', and 'Decrease in other activities' showed moderately strong association with the psychiatric comorbidity as a whole $(\phi=0.28)$ and with the internalizing disorders with Cramer's Vassociations $(\phi)$ 0.27, 0.23 and 0.23 , respectively. Although weak, the externalizing disorder showed association with 'Jeopardizing relationship/career' $(\phi=0.17)$ and 'Loss of Control' ( $\phi=0.16)$ (Table 5).

\section{DISCUSSION}

Our result revealed that Internet gaming is a very popular recreational activities in ROK adolescents. Since the confucian Korean culture traditionally values academic attainment very highly, the fact that almost $80 \%$ of students playing internet games with more than 2 hours of average daily gaming time was unexpected. Internet gaming is a popular recreation in these days, yet such high rate was not anticipated results. Since our participants were recruited from schools but not from online sources, which may include high risk group, these findings were surprising. Yet, only 16 students (4.8\%) were severe enough to be clinically diagnosed as IGD. This prevalence was 
Table 5. Association between IGD symptoms and psychiatric comorbidity among positive students ( $N=69)$

\begin{tabular}{lccc}
\hline & Overall Psychiatric comorbidities & Internalizing disorders & Externalizing disorders \\
\hline Preoccupation & 0.28 & 0.27 & 0.09 \\
Withdrawal & 0.28 & 0.23 & 0.14 \\
Tolerance & 0.01 & 0.09 & 0.14 \\
Loss of control & 0.14 & 0.04 & 0.16 \\
Decrease in other activities & 0.28 & 0.23 & 0.14 \\
Persistent use despite negative results & 0.05 & 0.09 & 0.04 \\
Deceiving & 0.03 & 0.03 & 0.01 \\
Escapism & 0.08 & 0.07 & -0.21 \\
Jeopardizing relationship/career & 0.15 & 0.05 & 0.17 \\
Craving & 0.07 & 0.01 & 0.09 \\
\hline
\end{tabular}

Values are Cramer's V $(\varphi)$. IGD: internet gaming disorder

comparable with the prevalence estimated in the general population..$^{29,30}$

The major finding of this study was that problematic gamers demonstrated different patterns of clinical manifestations by different levels of severity in gaming problems. The univariate curves made the hierarchic presentation of IGD criteria more evident.

Two IGD symptoms of 'Escapism' and 'Jeopardizing relationship/career' showed accelerating curvilinear lines, meaning that these IGD criteria were more frequent in severe subjects. Thus, the two symptoms may indicate more severe behavioral addiction in gaming and we argue that whenever encountering potential IGD patients with 'Escapism' or 'Jeopardizing relationship/career', more clinical attention is required in both diagnostic and therapeutic approaches.

'Loss of Control', 'Decrease in other activities', 'Persistent use despite Negative results' and 'Craving' demonstrated linear relationships with increasing gaming severity, suggesting a kind of dose-dependent pattern of clinical manifestations according to IGD severity. 'Decrease in other activities' was also the criterion that the addicted group (50.0\%) differed significantly from the moderate risk group (14.3\%). Therefore, this criterion could be a key screening question in detecting IGD.

On the other hand, 'Preoccupation, 'Withdrawal', 'Deceiving' and 'Tolerance' were plotted as decelerating curvilinear lines. Decelerating curvilinear lines mean that the symptoms are more prevalent among the low severity groups. Such 'lowthreshold' symptom could be a prevalent phenomenon but may not necessarily be an alarming sign of IGD by itself alone. However, an exception could be applied to the IGD criterion of 'Withdrawal'.

When compared to the moderate risk group (19.1\%), 'Withdrawal' tended to appear more often in the addicted group (58.3\%). The lack of statistically significance may owe to its uncommonness as it was the third infrequent symptom in overall (20.3\%) after 'Jeopardizing relationship/career' (17.4\%) and 'Deceiving' (18.8\%). Although not a frequently displayed symptom, the 'Jeopardizing relationship/career' symptom tended to represent the most severely addicted group. Thus, presence of this particular symptom warrants further exploration on the matters of gaming and hence providing more intensive treatment.

Another interesting finding of this study was that the morphology of the best fitting curve for the 'Tolerance' criterion was close to a flat shape. Moreover, 'Tolerance' did not display much meaningful associations with any other IGD criteria by Cramer's V association. Together with the outlier-like position revealed in the PCA as a solitary component of its own, this put a serious question mark on 'Tolerance' as a valid diagnostic criterion of IGD. The peerless position of 'Tolerance' may not necessarily represent its uniqueness but may rather indicate its failure as a diagnostic criterion in reflecting the true pathology IGD. The proposed DSM-5 IGD criteria of 'Tolerance' and 'Withdrawal' had been criticized or not regarded as auniversal feature. ${ }^{31,32}$ Our findings strongly support the developing ICD11 for not including 'Tolerance' as an essential element in diagnosing IGD.

As well as including 'Tolerance' in IGD with no clear empirical evidence, the currently proposed IGD criteria could be also criticized for omitting 'Craving', a traditionally important concept in addiction. 'Craving' previously demonstrated higher positive predictive rate (91.4\%) for IGD than other proposed criteria such as 'Preoccupation' (90\%), 'Withdrawal' (83.3\%) or 'Escapism' (85.2\%). ${ }^{33}$ Our result demonstrated that 'Craving' may discriminate the moderate risk group from mild risk group and has a linear relationship in the univariate curve, increasing its prevalence with heightening IGD severity. Thus, this finding highlights the potentialclinical utility of 'Craving' in IGD and indicates further investigations for its value in the IGD assessment. 
For the analysis of association between the IGD criteria, 'Preoccupation' demonstrated associations with 'Loss of Control' and 'Decrease in other activities.' 'Loss of Control' showed associations with 'Deceiving' and 'Persistent use despite Negative results'. 'Decrease in other activities' displayed associations with 'Deceiving, 'Persistent use despite Negative results', 'Craving, 'Withdrawal' and 'Jeopardizing relationship/career'. Apart from 'Decrease in other activities', 'Jeopardizing relationship/career' was associated with 'Deceiving' and 'Persistent use despite Negative results'.

'Preoccupation' was prevalent in the groups with lower severity and had been postulated as a beginning process of gaming addiction. ${ }^{34}$ The results of varying distribution of IGD criteria across different severity groups their associated patterns made the authors to hypothesize that preoccupation to gaming leads to impaired control to cut down gaming and diminished interest to other activities. These would, in turn, contribute to persistent gaming despite negative outcomes and make the gamers to lie about their addictive behavior in attempt to cover up their problems. Craving, under the influence of withdrawal, may further strengthen the diminished interest to daily activities and persistent gaming despite negative outcomes. However, when the efforts to compensate their addictive behavior (e.g., deceiving) fail, ones may ultimately face significant losses in interpersonal relationships or career opportunities. Although this hypothesized temporal development of IGD pathology may sound convincing, this cannot be verified in this cross-sectional outcomes and requires well-designed longitudinal studies to test whether IGD progress in such sequential manner.

Even though the above explanation for the pathogenesis of IGD from low-threshold to high-threshold symptoms in a sequential manner may sound convincing, this hypothesis has to be remain as a mere speculation since we cannot attribute causality for one IGD symptom to another cue to the cross-sectional nature of our current investigation. Such inference on the course of IGD pathogenesis can only be derived from carefully designed longitudinal studies. Nevertheless, we anticipate that the ongoing cohort study (iCURE) will hopefully broaden our knowledge regarding the natural course of IGD.

The fact that other individual, environmental and gamerelated risk factors, which may influence over the onset and clinical presentation of IGD such as temperaments, parentchild or peer relationships, game genres, respectively, were not analyzed is also a limitation in this study. Future studies would be followed to examine potential risk or protective factors with more data collection. Although we tried our very best to minimize sample bias in the study, another limitation would be from confounding factors arising from the study sample. The entire study sample was comprised of student.
While this homogeneity may provide more accurate evaluation for the increasing gaming problems in the adolescents, this also may limit generalizability to general population since students are not free to play games in the school hours and have different levels of parental guidance. Other limitations on generalizability may arise due to the fact that all participants were Koreans residing in Seoul metropolitan area. This may limit the application of our study results to people living in rural regions or other nations.

To the best of our knowledge, this investigation was the first study that ever attempted to explore the association of IGD severity with comorbid internalizing or externalizing problems by formal psychiatric diagnosis. Unless treated separately, the influence of the two distinct groups on IGD may disappear due to a mixed effect. While comorbid internalizing disorders demonstrated an accelerated pattern in the univariate curve, externalizing disorders demonstrated a deceleratedpattern in accordance to the increasing severity of gaming problems. This finding suggest that ADHD or other conditions with difficulties in executive control, may actually lower the hurdle for the vulnerable ones to display problematic gaming pattern at relatively earlier stage of IGD. In a study of drug naïve children having both ADHD and video gaming problems, 8 weeks of methylphenidate treatment improved the measure related to the Internet addiction and spending time as well as improving the attention problems. ${ }^{35}$ Together with our finding, this implicates that treating such predisposing comorbid conditions may increase resistance to the onset of IGD or facilitate the recovery process. Nonetheless, further investigations are required for confirmation.

Even though 'Escapism' displayed an accelerated curve in our analysis, this phenomenon may owe, at least in part, to the similar accelerated pattern of internalizing disorders (Figure 2). Therefore, more prospective studies are also needed to illuminate the relationships between internalizing disorders like depression and 'Escapism', the criteria of utilizing games as a means for mood modification and to ultimately reveal the relationship of internalizing disorders with IGD.

The authors previously proposed the typology of IGD as the impulsive/aggressive, the emotionally vulnerable, the socially conditioned subtype. ${ }^{36}$ This study's findings demonstrated that theclinical picture may vary by the externalizing disorders (the impulsive/aggressive type) and the internalizing disorders (the emotionally vulnerable type) in terms of severity and associated symptoms. This finding may add some important insights about the varying effects of psychiatric comorbidities on IGD and its typology.

To verify whether a particular symptom indicates a prodromal feature on its way to developing IGD or is just a symptom clustered in accordance to corresponding severity, more stud- 
ies should be followed in future. Our results revealed that there is a hierarchic order in IGD criteria and certain symptoms like 'Decrease in other activities' and 'Jeopardizing relationship/career' may represent a higher severity of IGD. Therefore, allocating more clinical resources to such phenomena seems just, instead of viewing all IGD symptoms equally.

\section{Acknowledgement}

This study was supported by a grant of the Korean Mental Health Technology R\&D Project, Ministry of Health \& Welfare, Republic of Korea (HM14C2603). We also appreciate Mi Sun Park M.S. for the statistical analysis support.

\section{REFERENCES}

1. Gross EF, Juvonen J, Gable SL. Internet use and well-being in adolescence. J Soc Issues 2002;58:75-90.

2. Subrahmanyam K, Kraut RE, Greenfield PM, Gross EF. The impact of home computer use on children's activities and development. Fut Child 2000;123-144.

3. (APA) APA. Diagnostic and Statistical Manual of Mental Disorders. Arlington, VA: American Psychiatric Publishing; 2013.

4. Gaming disorder (ICD-11 Beta Draft). Available at: http://apps.who. int/classifications/icd11/browse/l-m/en\#/http\%3a\%2f\%2fid.who.int\% 2ficd\%2fentity\%2f1448597234. Accessed June 17, 2016.

5. International_Telecommunication Union. ICT Facts and Figures 2015. Geneva: International Telecommunication Union; 2015.

6. Gauffin K, Vinnerljung B, Hjern A. School performance and alcoholrelated disorders in early adulthood: a Swedish national cohort study. Int J Epidemiol 2015;44:919-927.

7. Imamura K, Kawakami N, Inoue A, Shimazu A, Tsutsumi A, Takahashi $\mathrm{M}$, et al. Work engagement as a predictor of onset of Major Depressive Episode (MDE) among workers, independent of psychological distress: a 3-year prospective cohort study. PLoS One 2016;11:e0148157.

8. Wang J. Work stress as a risk factor for major depressive episode(s). Psychol Med 2005;35:865-871.

9. Wei HT, Chen MH, Huang PC, Bai YM. The association between online gaming, social phobia, and depression: an internet survey. BMC Psychiatry 2012;12:92.

10. Gentile DA, Choo H, Liau A, Sim T, Li D, Fung D, et al. Pathological video game use among youths: a two-year longitudinal study. Pediatrics 2011;127:e319-329.

11. Achab S, Nicolier M, Mauny F, Monnin J, Trojak B, Vandel P, et al. Massively multiplayer online role-playing games: comparing characteristics of addict vs non-addict online recruited gamers in a French adult population. BMC Psychiatry 2011;11:144.

12. Kim NR, Hwang SS, Choi JS, Kim DJ, Demetrovics Z, Kiraly O, et al. Characteristics and psychiatric symptoms of internet gaming disorder among adults using self-reported DSM-5 criteria. Psychiatry Investig 2016;13:58-66.

13. Dalbudak E, Evren C. The relationship of Internet addiction severity with Attention Deficit Hyperactivity Disorder symptoms in Turkish University students; impact of personality traits, depression and anxiety. Compr Psychiatry 2014;55:497-503.

14. Wang CW, Chan CL, Mak KK, Ho SY, Wong PW, Ho RT. Prevalence and correlates of video and internet gaming addiction among Hong Kong adolescents: a pilot study. Scientific World Journal 2014;2014: 874648 .

15. Muller KW, Janikian M, Dreier M, Wolfling K, Beutel ME, Tzavara C, et al. Regular gaming behavior and internet gaming disorder in European adolescents: results from a cross-national representative survey of prevalence, predictors, and psychopathological correlates. Eur Child Adolesc Psychiatry 2015;24:565-574.
16. Orsolya Kiraly KN, Mark D. Griffiths and Zsolt Demetrovics. Behacioral Addictions: Criteria, Evidence and Treatment. San Diego, CA: Elsevier; 2014.

17. Clark CB, Zyambo CM, Li Y, Cropsey KL. The impact of non-concordant self-report of substance use in clinical trials research. Addict Behav 2016;58:74-79.

18. Miller P, Curtis A, Jenkinson R, Droste N, Bowe SJ, Pennay A. Drug use in Australian nightlife settings: estimation of prevalence and validity of self-report. Addiction 2015;110:1803-1810.

19. Toce-Gerstein M, Gerstein DR, Volberg RA. A hierarchy of gambling disorders in the community. Addiction 2003;98:1661-1672.

20. Khazaal Y, Achab S, Billieux J, Thorens G, Zullino D, Dufour M, et al. Factor structure of the internet addiction test in online gamers and poker players. JMIR Ment Health 2015;2:e12.

21. Sanders JL, Williams RJ. Reliability and validity of the behavioral addiction measure for video gaming. Cyberpsychol Behav Soc Netw 2016;19:43-48.

22. Kim YS, Cheon KA, Kim BN, Chang SA, Yoo HJ, Kim JW, et al. The reliability and validity of Kiddie-Schedule for Affective Disorders and Schizophrenia-Present and Lifetime Version- Korean version (KSADS-PL-K). Yonsei Med J 2004;45:81-89.

23. Krueger RF. The structure of common mental disorders. Arch Gen Psychiatry 1999;56:921-926.

24. Cramer P. Change in children's externalizing and internalizing behavior problems: the role of defense mechanisms. J Nerv Ment Dis 2015;203:215-221.

25. Fisher BW, Gardella JH, Teurbe-Tolon AR. Peer cybervictimization among adolescents and the associated internalizing and externalizing problems: a meta-analysis. J Youth Adolesc 2016;45:1727-1743.

26. Lande MB, Adams H, Falkner B, Waldstein SR, Schwartz GJ, Szilagyi PG, et al. Parental assessments of internalizing and externalizing behavior and executive function in children with primary hypertension. J Pediatr 2009;154:207-212.

27. Verona E, Sachs-Ericsson N, Joiner TE Jr. Suicide attempts associated with externalizing psychopathology in an epidemiological sample. Am J Psychiatry 2004;161:444-451.

28. Compulsive Behavior. Available at: https://meshb.nlm.nih.gov/record/ ui?ui=D003192. Accessed August 13, 2016.

29. Rehbein F, Kliem S, Baier D, Mossle T, Petry NM. Prevalence of Internet gaming disorder in German adolescents: diagnostic contribution of the nine DSM- 5 criteria in a state-wide representative sample. Addiction 2015;110:842-851.

30. Papay O, Urban R, Griffiths MD, Nagygyorgy K, Farkas J, Kokonyei G, et al. Psychometric properties of the problematic online gaming questionnaire short-form and prevalence of problematic online gaming in a national sample of adolescents. Cyberpsychol Behav Soc Netw 2013; 16:340-348.

31. Kardefelt-Winther D. Meeting the unique challenges of assessing internet gaming disorder. Addiction 2014;109:1568-1570.

32. Kaptsis D, King DL, Delfabbro PH, Gradisar M. Withdrawal symptoms in internet gaming disorder: a systematic review. Clin Psychol Rev 2016;43:58-66.

33. Ko CH, Yen JY, Chen SH, Wang PW, Chen CS, Yen CF. Evaluation of the diagnostic criteria of Internet gaming disorder in the DSM-5 among young adults in Taiwan. J Psychiatr Res 2014;53:103-110.

34. Young K. Understanding online gaming addiction and treatment issues for adolescents. Am J Fam Ther 2009;37:355-372.

35. Han DH, Lee YS, Na C, Ahn JY, Chung US, Daniels MA, et al. The effect of methylphenidate on Internet video game play in children with attention-deficit/hyperactivity disorder. Compr Psychiatry 2009;50:251-256.

36. Lee SY, Lee HK, Choo H. Typology of internet gaming disorder and its clinical implications. Psychiatry Clin Neurosci 2016 [Epub ahead of print]. 